\title{
Kernos
}

Revue internationale et pluridisciplinaire de religion grecque antique

$15 \mid 2002$

Varia

\section{De quelques thématiques déterminantes relatives à la religion grecque antique}

\section{Evanghelos A. Moutsopoulos}

\section{(apenEdition}

\section{Journals}

Édition électronique

URL : http://journals.openedition.org/kernos/1358

DOI : 10.4000/kernos.1358

ISSN : 2034-7871

Éditeur

Centre international d'étude de la religion grecque antique

Édition imprimée

Date de publication : 1 janvier 2002

ISSN : 0776-3824

Référence électronique

Evanghelos A. Moutsopoulos, «De quelques thématiques déterminantes relatives à la religion grecque antique », Kernos [En ligne], 15 | 2002, mis en ligne le 21 avril 2011, consulté le 22 avril 2019. URL http://journals.openedition.org/kernos/1358; DOI : 10.4000/kernos.1358 


\section{De quelques thématiques déterminantes}

\section{relatives à la religion grecque antique*}

Dans mon discours d'ouverture prononcé lors du Premier Congrès du CIERGA (alors CERGA) ${ }^{1}$, je traçais les grandes lignes d'orientation de la recherche dans ce domaine et en définissais les thématiques principales, sinon exclusives. Ce processus programmatique aurait pu sembler, à l'époque, quelque peu arbitraire, étant établi d'avance et d'en haut. Je constate aujourd'hui que mes conceptions et prévisions ont été pour le moins fondées, puisque tout au long des quatorze années qui séparent ce Premier Congrès, tenu à Athènes, de celui de Rhodes, qui s'ouvre aujourd'hui, la permanence des thématiques entrevues à l'époque se voit, pour ainsi dire, confirmée après coup et d'en-bas, et que la quasi-totalité de ces thématiques se reflètent dans les titres mêmes des communications proposées.

Telles qu'elles se sont imposées au cours des années, les grandes thématiques ainsi construites se sont révélées déterminantes et incontournables pour les participants aux Congrès du CIERGA, et ont permis de forger une série de catégories auxquelles tout titre de communication est, comme naturellement, subsumable. Il suffit de parcourir les titres des communications qui ont été soumis au comité d'organisation de notre Congrès pour s'en convaincre. Sans soutenir que la liste de ces catégories, examinées par la suite, soit rigoureusement et définitivement exhaustive, du fait que rien n'est à exclure dans l'avenir, je constaterai toutefois qu'elle tend à devenir classique.

Cette liste comprend en principe une douzaine de catégories bien distinctes dont les domaines sont cependant loin d'être incompatibles les uns par rapport aux autres; autrement dit, il n'est pas improbable qu'ils se prêtent à une sorte d'osmose, en raison de la complexité des sujets qui s'y réfèrent. Mais toutes ces catégories se projettent sur le fond commun que constitue la religion grecque antique ${ }^{2}$. Elles concernent respectivement les mythes, les cultes et les rites, auxquels il convient de rattacher la magie et la divination,

\footnotetext{
* Discours d'ouverture du VIII ${ }^{e}$ Congrès du CIERGA (Rhodes, mai 2001).

1 Cf. E. Moutsopoulos, "Vers une approche pluridisciplinaire de la religion grecque antique", Kernos 1 (1988), p. 105-110.

2 Tout comme les diverses catégories esthétiques se projettent sur fond de beauté, alnsi que je l'ai montré dans mon livre sur les catëgories esthétiques. Introduction à laxiologie de l'objet esthëtique, Athènes, 1970, 1996², p. 15.
} 
ainsi que les disciplines auxiliaires, telle que l'astronomie. Elles incluent également les institutions et la conception philosophique de la religion et de ses pratiques aussi bien à l'âge classique qu'au début de l'ère chrétienne, notamment (dans ce dernier cas) en ce qui concerne le problème des rapports entre la foi et la raison. Enfin, l'apport de l'archéologie (en association avec l'histoire) entraîne une meilleure connaissance des informations dispensées par les sources.

Sans détailler les thématiques des communications présentées aux congrès précédents, faciles à dépister rien qu'en parcourant les volumes respectifs de Kernos, il s'avère que la liste des communications du Congrès de Rhodes les résume en tant qu'aboutissement, ne serait-ce que provisoire, de nos efforts communs. Ces communications ont été groupées quasi automatiquement autour des axes désormais traditionnels. Le mythe d'abord. Il n'existe point de religion qui ne s'appuie sur un mythe ou sur un ensemble de mythes dont les uns ne sont que le développement des autres. Claude LéviStrauss a bien montré la structure quasi musicale des mythes fondamentaux et de leurs compléments, qui se fondent en un ensemble solidement lié $e^{3}$.

De son côté, et en suivant une voie analogue, Lambros Couloubaritsis, récemment élu membre correspondant de l'Académie d'Athènes, a établi, dans ses nombreux travaux originaux, que le mythe, loin de représenter une formation toute faite, est une entité en formation perpétuelle érigée en fonction des diverses mutations qui interviennent dans les sociétés concernées, et que lui-même fonctionne, au-delà d'une conception dynamique de la réalité qu'il représente, comme un jeu. De ce fait, Couloubaritsis propose une nouvelle méthode de lecture des mythes, aux termes de laquelle leur message, qui en fait de véritables logoi, ne se réduit pas simplement à « un discours sauvegardant le patrimoine culturel et ritualisé ", mais renferme « des potentialités cognitives importantes et même affectives $»^{4}$.

J'ajouterai que, dans le même ordre d'idées, ainsi que je l'ai souvent affir$\mathrm{me}^{-5}$, le mythe fonctionne comme l'expression de l'acceptation axiologique d'une situation effective, mais susceptible d'être modifiée, voire renversée, dans le temps. Dans ce cas, le mythe qui exprime cette'situation se voit réajuster en vue de refléter, en s'y adaptant, l'acceptation d'une nouvelle phase de la réalité, une fois consolidée, et ainsi de suite, tout en retenant des éléments de sa structure antérieure. D'où la complexité de la stratification mythique qu'il suffit d'analyser, de " déstructurer », pour parler dans un langage à la mode, afin d'y retrouver le noyau originel du mythe, noyau qui conduira à la réalité originelle qui en a déterminé la naissance.

3 Cl. LÉvi-Strauss, Mytbologiques. 1. Le cru et le cuit, Paris, Plon, 1962, p. 11 sq.

4 L. Couloubaritsis, Mythe et philosophie chez Parménide, Bruxelles, Ousia, 1986; Aux origines de la pensée européenne. De la pensée archalque au néoplatonisme, Bruxelles, $2000^{3}$ (Le point philosopbique); La philosopbie et la question du mytbe, Bruxelles, 1997.

\footnotetext{
5 Par ex. dans Philosophes de l'Ege, Ahenes, Fondation de l'Egee, 1991, p. 19-23.
} 
Dans ces conditions, les religions pourraient être interprétées comme des conceptions, à la fois statiques et dynamiques, dérivées des mythes. Elles supposent des mythes cosmogoniques et des systèmes comportant une hiérarchie de valeurs que fécondent et que cherchent à consolider des conceptions fondées sur des spéculations d'ordre astronomique ou autre, soumises, par exemple, à des idées telle celle de péríodicité, d'où l'importance de disciplines comme l'astronomie, pour sceller la valeur du mythe fondateur autant que pour en faciliter l'interprétation de notre part.

C'est sur ces structures mythiques que se greffent, tour à tour, cultes et rites : cultes de dieux ou de héros divinisés, souvent érigés en principes à atteindre, en souvenir, et selon un procédé analogue d'abstraction relatif à des forces surnaturelles, par exemple la mana, puissance vitale, auxquelles se sont originairement référées les pratiques magiques accompagnant obligatoirement les manifestations de la mentalité « primitive », « mythique » ou " archaïque », telle que l'ont respectivement envisagée Lucien Lévy-Bruhl ${ }^{6}$ ou Jean Cazeneuve ${ }^{7}$, bref "pré-aristotélicienne », dirions-nous. Ces cultes, généralisés ou strictement locaux, sont, en fait, des célébrations de divinités résultant souvent de contractions, d'associations ou de fusions de plusieurs divinités individuelles qui se sont superposées dans le temps. À peine consolidés, ces cultes s'institutionnalisent pour rejoindre les institutions sociales et, ajouterai-je, économiques qui en ont favorisé l'éclosion, en s'y intégrant.

Les rites, quant à eux, sont des manifestations précises et spécifiques (mais réductibles à une typologie) de chacun des cultes envisagés, et qui expriment la nécessité de permanence des cultes en question, moyennant leur invariabilité, d'où leur transformation fréquente en rituels. Il est important de constater l'intégration organique des rites et de leurs aspects ritualisés dans des institutions, déjà existantes ou réformées en fonction de ces rites une fois établis. Une dialectique polymorphe s'installe entre les sociétés ellesmêmes et leurs divers modes d'extériorisation cultuelle et rituelle, voire culturelle. N'est-ce pas sur le tard seulement que la tragédie grecque, par exemple, se vit détacher du culte de Dionysos pour s'incorporer, en tant que véritable institution culturelle, dans l'ensemble des institutions athéniennes ${ }^{8}$ ?

Magie et divination occupent chacune dans ce contexte une place particulière. Incontestablement tributaires l'une de l'autre à l'origine, leur développement et leur imposition ont été rendus nécessaires en vue de capter des puissances bénéfiques et de neutraliser des puissances maléfiques. Si cette fonction appartient notamment à la magie, celle, plus caractéristique, de la divination est de déterminer dans quelle direction adéquate il convient d'exercer les pratiques magiques; d'où la double attribution du personnage fréquemment chargé de remplir à la fois les deux dignités de sorcier et de

La mentalité primitive, Paris, $1960^{5}$.

7 La mentalité archaique, Paris, 1961.

8 Cf. déjà L. Drubner, Attische Feste, Berlin, 1932. 
devin, qui néanmoins demeurent bien distinctes à l'intérieur des conduites religieuses.

C'est ici qu'intervient la philosophie à proprement parler : le logos philosophique fonctionne selon les mêmes principes que le logos mythique, tels les principes d'identité ou de causalité, mais en diffère en tant qu'il opère selon des critères très rigoureux : principes de contradiction ou du tiers exclu, voire exclusion obligatoire de toute référence à des causes premières. D'où son intérêt primordial pour la compréhension, par opposition au mythe et à ses développements : cultes, rites etc, dans un souci d'en interpréter et d'en apprécier la signification, sans en justifier la réalité pour autant. À commencer par la critique de Xénophane, reprise par Platon qui, cependant, n'hésite pas à adopter certaines pratiques cultuelles dans son souci de demeurer fidèle à l'enseignement de Damon d'Oa, aux termes duquel un état ne peut prétendre à la continuité que s'il favorise l'invariabilité de ses institutions et de sa culture $^{9}$. Tout en acceptant partiellement les thèses de Platon, Aristote se garde bien de concéder autant de droits que lui à l'irrationnel ${ }^{10}$. Trois références à Platon, une à Aristote et une au stoïcisme seront présentées à notre Congrès, de même qu'une référence à la condamnation du prétendu athéisme chrétien.

Il s'agit, on le constate aisément, d'un certain nombre d'attitudes divergentes, mais tout compte fait, similaires quant à leur origine et leur finalité. La catégorie relative au rapport entre foi et raison délimite une thématique pertinente illustrée déjà par Xénophane, et qui atteindra le point culminant de son importance chez les Pères de l'Église. Toutefois, assistée de l'histoire qui, de son côte, sert à définir strictement l'enchaînement des événements, et en collaboration avec elle, l'archéologie, discipline historique, offre à nos travaux une catégorie thématique distincte et autonome à laquelle l'archéométrie apporte indubitablement un soutien de poids. Comme telle, l'archéologie est à même d'éclairer d'un jour nouveau, ainsi que je l'ai déjà affirmé, les témoignages relatifs à la religion grecque antique dispensés par les sources littéraires et épigraphiques, en vertu de son propre apport, à savoir l'ensemble des objets, rituels et autres, mis à jour à la faveur de fouilles conduites dans des sites appropriés. Dès lors, comment s'étonner du nom de «Kernos », donné à notre revue officielle et accompagné d'une représentation de cet objet, logotype de notre Association?

Je n'ai fait que mentionner, dans ce qui précède, les diverses catégories thématiques de nos recherches, en essayant de les élucider. Ce faisant, je me suis aperçu que je venais d'énoncer tout un programme d'activités d'érudi-

9 Cf. E. Moutsopoulos, "L'idée de kalokagatbia et sa fonction éthique et esthétique en Occident", Poïésis et technè. 2. Instauration et vibration, Montréal, Montmorency, 1994, p. 219-238, notamment p. 221-223.

10 Cf. $i d$., "Culture musicale, culture morale et politique chez Aristote", in La società civile e la societá politica nel pensiero di Aristotele, Roma, Centro Intern. di Filosofia Antica "Antonio Jannone", 1998, p. 101-114. 
tion, qui échoient au CIERGA, et qui correspondent précisément à ses buts. Les domaines d'érudition ainsi définis sont, je le répète, hautement et fidèlement respectés et cultivés depuis les origines de notre Association. Ils illustrent la pluridisciplinarité de notre entreprise telle qu'elle fut conçue à ses débuts et marquent la constance avec laquelle elle s'est développée au cours des années écoulées, pour s'affirmer en tant que véritable institution internationale. Par ses thématiques particulières, le Congrès de Rhodes ne fait que confirmer la réussite de cette entreprise et justifier les vues que j'avais exposées lors de sa naissance.

E. Moutsopoulos 